\title{
Grain Boundaries across Length Scales; Correlating SEM, Aberration- Corrected TEM Orientation Imaging and Nanospectroscopy
}

\author{
W.J. Bowman ${ }^{1}$, A. Darbal ${ }^{2}$, M. Kelly ${ }^{3}$, G.S. Rohrer ${ }^{3}$, C.H. Hernandez ${ }^{1}$, K. McGuinness ${ }^{1}$, P.A. \\ Crozier $^{1}$
}

1. School for the Engineering of Matter, Transport and Energy, Arizona State University, Tempe Arizona 85287-6106, USA

2. AppFive LLC, Tempe, Arizona, USA

3. Materials Research Science and Engineering Center, Carnegie Mellon University, 5000 Forbes Avenue, Pittsburgh, PA 15213, USA

Grain boundaries significantly influence the behavior of a huge range of materials; however, their small size $(\sim 1 \mathrm{~nm}$ structural width) and significant variety make them difficult to characterize, fully describe, and ultimately optimize. Statistical methods such as stereological analysis coupled with SEM orientation imaging via electron back scatter diffraction (EBSD) which typically samples $>50,000$ boundaries, are powerful techniques for quantifying microscopic grain boundary parameters such as the crystallographic plane distribution and misorientation texture [1]. Conversely, TEM and STEM atomic resolution imaging and spectroscopies have proven invaluable in elucidating nanoscale boundary properties including local atomic structure and elemental composition [2]. However, correlating such micro- and nano-scale observations has been challenging due to the difficulty of unambiguously determining the statistical relevance of grain boundaries observed in the TEM.

Recently-commercialized precession-enhanced nanobeam diffraction (PEND) instrumentation and techniques have made possible routine orientation imaging in the aberrationcorrected TEM (AC-TEM) [3]. This mesoscale technique facilitates the location and identification of statistically relevant grain boundaries in a TEM specimen that can then be targeted for in-depth analysis via high resolution imaging and/or nanospectroscopy. This capability bridges the gap between microscopic SEM measurements and nanoscopic and ACSTEM characterizations to offer a more complete and contiguous understanding of grain boundary properties across a considerable range of length scales.

Here, we employ a suite of techniques including SEM EBSD, AC-TEM PEND and ACSTEM EELS and EDX to correlate data sets acquired across micron, meso and nanometer length scales from ceria-based oxygen-conducting ceramics. We present findings and results based on stereological analysis performed to characterize microscopic grain boundary parameters derived from SEM EBSD data (fig. (a)) such as plane distribution and misorientation texture. We describe the use of these parameters, such as boundary misorientation angle distribution (e.g. fig. (b)), to relate data acquired from SEM and AC-TEM orientation imaging techniques. Figure (c) presents a representative orientation image acquired with an AC-TEM equipped with an ASTAR PEND system. We discuss correlation of this mesoscale boundary characterization with SEM EBSD results. Furthermore, we present image and nanospectroscopic data acquired from the PEND specimens using an AC-STEM equipped with EELS and EDX. Figure (d) shows ADF AC-STEM images of boundaries selected for analysis based on PEND results. The images presented here demonstrate the diversity in atomic structure found in the observed boundaries. AC-STEM EELS spectra acquired on and off the boundaries in fig. (d), at the locations highlighted, are presented in fig. (e). Energy-loss spectra show variation in cation concentrations 
with distance from the boundary core, as well as changes in near-edge fine structure, indicating spatial dependence of the cation oxidation states likely stemming from local variations in oxygen non-stoichiometry.

\section{References and acknowledgements}

[1] L. Helmick et al. Int. J. Appl. Ceram. Technol., 8 [5] 1218-1228 (2011)

[2] S.J. Pennycook \& P.D. Nellist. Scanning Transmission Electron Microscopy, Springer

[3] A.D. Darbal et al. Microsc. Microanal. 19, 111-119 (2013)

[4] C.A.H. and K.M. wish to thank the Fulton Undergraduate Research Initiative at ASU for generous financial support throughout this work. W.J.B. would like to acknowledge the National Science Foundation's Graduate Research Fellowship (DGE-1211230) for continued financial support. Finally, we gratefully acknowledge support of NSF grant DMR-1308085 and ASU's John M. Cowley Center for High Resolution Electron Microscopy.

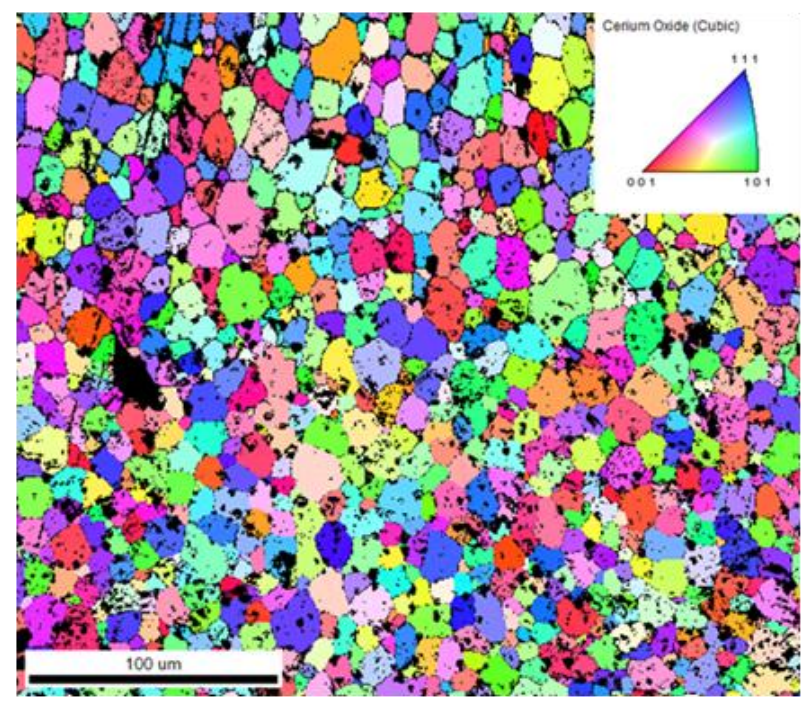

(a)

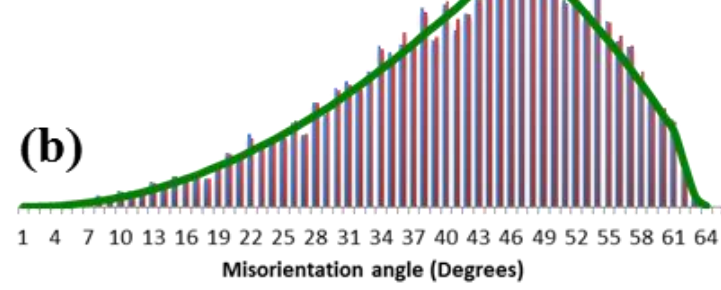

(d)

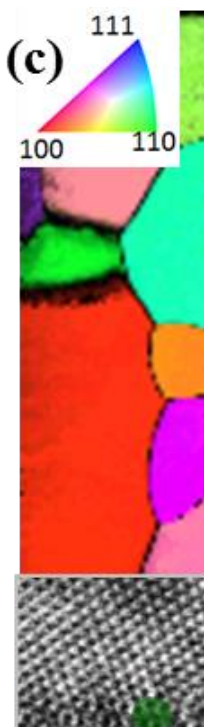

(e)
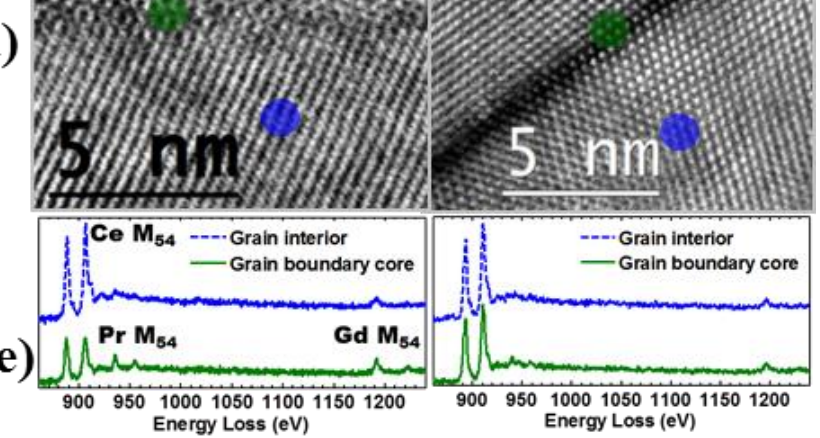

Fig. a. SEM EBSD inverse pole figure of Ca-doped ceria.

Fig. b. Misorientation angle distribution determined from SEM EBSD.

Fig. c. AC-TEM PEND inverse pole figure of $\mathrm{Gd} / \mathrm{Pr}$-doped ceria.

Fig. d. AC-STEM ADF images of grain boundaries selected from figure (d).

Fig. e. AC-STEM EELS spectra acquired on and off of the grain boundaries in figure (e). 